\title{
ON THE DIRECT DETERMINATION OF THE EIGENMODES OF FINITE FLOW-STRUCTURE SYSTEMS
}

\author{
By Mark W. Pitman \& Anthony D. Lucey \\ Fluid Dynamics Research Group, Curtin University of Technology, Australia \\ E-mail: M.Pitman@curtin.edu.au; A.Lucey@curtin.edu.au
}

A new method for directly determining the eigenmodes of finite flow-structure systems is presented using the classical problem of the interaction of a uniform incompressible flow with a flexible panel, held at both ends, as an exemplar. The method is a hybrid of theoretical analysis and computational modelling. This method is contrasted with Galerkin and travelling-wave methoda that are most often used to study the hydro-elasticity of such systems. The new method does not require an a priori approximation of perturbations via a finite sum of modes. Instead, the coupled equations for the wall-flow system are used to derive a single matrix equation for the system that is a second-order differential equation for the panel-displacement variable. This is achieved in this exemplar by applying a combination of boundaryelement and finite-element methods to the discretised system. Standard state-space methods are then used to extract the eigenmodes of the system directly. We present results for the stability of the case of an unsupported flexible plate, elucidating its divergence and flutter characteristics, and the effect of energy dissipation in the structure. We then present results for the case of a spring-backed flexible plate showing that its motion is dominated by travelling waves. Finally, we illustrate the versatility of the approach by extracting the stability diagrams and modes for a panel with spatially varying properties and a panel with non-standard boundary conditions. In doing so, we show how spatial inhomogeneity can modify the energy exchanges between flow and structure, thereby introducing a single-mode flutter instability at pre-divergence flow speeds.

Keywords: Flow-structure interaction, Panel instability, Eigen-analysis, Hybrid computational-theoretical methods

\section{Introduction}

This paper re-visits the most fundamental problem in aero/hydro-elasticity, depicted in Figure 1, wherein a uniform incompressible flow interacts with a flexible wall mounted over a rigid baffle. In the absence of a spring foundation and wall damping, the wall comprises a simple elastic plate. At a sufficiently high ratio of fluid-flow loading to plate stiffness, $\Lambda^{F}=\rho U_{\infty}^{2} L^{3} / B$, where $\rho$ and $U_{\infty}$ are the fluid density and speed and $B$ and $L$ are the panel's flexural rigidity and length, motions of the plate become linearly unstable through divergence instability. At higher flow speeds the panel undergoes divergence recovery and soon thereafter a flutter instability sets in. Thus, this problem has commonly become known as that of 'panel flutter'. 


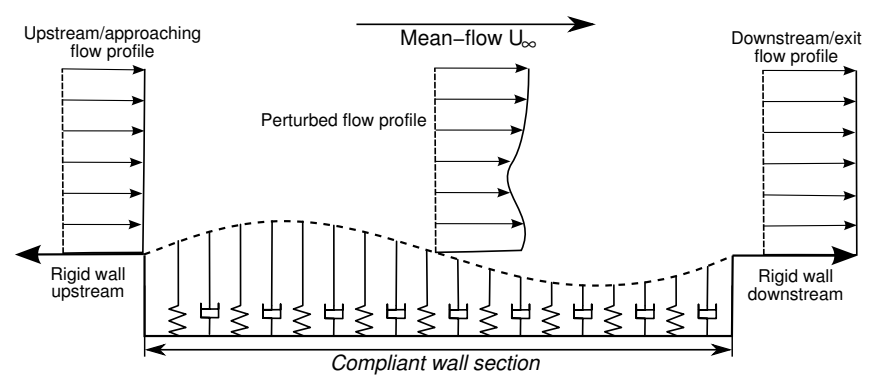

Figure 1. Schematic of the flow-structure system studied; the spring and dashpot foundations are absent for an unsupported elastic plate

The standard Galerkin method applied to such problems (for example, see Dugundji et al. 1963, Ishii 1965, Weaver \& Unny 1971, Ellen 1973, Garrad \& Carpenter 1982, Lucey \& Carpenter 1993) requires that the deformation of the flexible panel is written as a sum of orthogonal functions, or eigenmodes, that satisfy the boundary conditions at the panel's ends. The functions most commonly used are the in-vacuo modes of the panel. Thus, for a simple plate with hinged edges, the (complex) deflection variable is written as

$$
\eta=\exp (s t) \sum_{n=1}^{\infty} A_{n} \sin \left(\frac{n \pi x}{L}\right),
$$

where the eigenvalue $s$ is complex to capture both oscillatory motion and temporal growth/decay and the coefficients $A_{n}$ may also be complex. To make the problem tractable, a finite set of $R$ in-vacuo modes is selected. In the coupled flow-structure system, the eigenmodes (and eigen-frequencies) can differ very markedly from the in-vacuo set. It is then required that this same selected set of $R$ in-vacuo modes can be combined to represent accurately the disturbances in the coupled flow-structure system; this can be achieved by increasing the number of modes included in the computation. For fluid-structure systems with simple geometric and/or material configurations this strategy is very effective as will be demonstrated in this paper.

The natural theoretical alternative to the Galerkin method for the problem at hand is to represent the plate deformation, and system perturbations, by a continuous spectrum of modes, hence the travelling-wave assumption that

$$
\eta=A \exp [\mathrm{i}(k x-\omega t)]
$$

where $k=2 \pi / \lambda$ is the wavenumber, $\lambda$ is the wavelength and $\omega$ is the complex frequency of system disturbances. This assumption is strictly only valid for a panel of infinite length because no account is taken for the end conditions of the panel. However, it yields predictions of divergence-onset flow speed that agree well with the Galerkin approach for a critical mode with $\lambda \ll L$ that is the case for more complex flexible-wall structures such as a spring-backed flexible plate; see Carpenter \& Garrad (1986) and Lucey et al. (2003) wherein the assumption of localised hydrodynamic influence holds. This relationship between local and global hydroelastic modes is explored for the related problem of a flexible fluid-filled pipe by Doaré \& de Langre (2002). The travelling-wave approach predicts that energy dissipation 
by the plate/wall is required to realize divergence instability. This is physically implausible because exactly at onset the divergence wave is static; this inconsistency has recently been explained by Peake (2004) and is shown to arise from the end effects that are absent in the travelling-wave models.

The closely related proper, initial-value, boundary-value problem, wherein disturbances develop from a localized source of excitation has been studied by BrazierSmith \& Scott (1984), Crighton \& Oswell (1991), Peake (1997) and Abrahams \& Wickham (2001) for infinitely long plates. Like the travelling-wave approach, this entails the extraction of valid poles (or solutions) governed by the system dispersion relation subject to the principle of causality. The numerical simulations of Lucey (1998) for the equivalent finite system again highlighted a marked difference from the predictions for infinite systems that do not incorporate plate end-conditions and, thereby, a discretisation of the eigenmode spectrum.

Numerical simulations such as those of Lucey \& Carpenter (1992) and Lucey (1998) are ideal for capturing the transient behavior of finite flow-structure systems in response to an initial, or continuing, applied excitation. However, they are less suited to identifying the long-time solution in terms of system eigenmodes. This limitation is overcome by the present work in which we fuse computational and theoretical methods to provide a direct evaluation of the eigenmodes of the coupled flow-structure system.

For flow-structure interactions, the approach presented in this paper should be considered complementary to the Galerkin and travelling-wave approaches discussed above, but is clearly more closely related to the Galerkin in that it predicts the global stability of a finite system. The accuracy of the method presented herein is dependent upon the number of collocation points, $N$, used in the discretisation of the system. Increasing $N$ then gives a monotonically reducing rate of convergence as the solution approaches being exact at the limit at $N=\infty$. The method is particularly useful for the study of non-homogeneous flow-structure systems. Below, we outline our new hybrid method as applied to the paradigmatic problem of Figure 1, presenting a fairly comprehensive description of the linear dynamics of the system. Thereafter we demonstrate its application to spatially inhomogeneous panel configurations for which we show how and why these are susceptible to a weak flutter-type instability in the pre-divergence range of flow speeds. In concluding, we also note that this new approach is equally applicable to any flow-structure system for which computational models can be used to reduce the coupled system to a single differential equation for the motion of the fluid-solid interface.

\section{System equations}

The small-amplitude motion of a thin elastic plate, supported by a spring foundation $K$, in the presence of a fluid flow is described by

$$
\rho_{m} h \frac{\partial^{2} \eta}{\partial t^{2}}+d \frac{\partial \eta}{\partial t}+B \frac{\partial^{4} \eta}{\partial x^{4}}+K \eta=-\Delta p(x, 0, t)
$$

where $\eta(x, t), \rho_{m}, h$ and $B$ are, respectively, the plate's deflection, density, thickness and flexural rigidity, while $p(x, y, t)$ is the unsteady fluid pressure. We include a dashpot-type damping term, with coefficient $d$, to model the effects of energy dissipation in the wall structure. While this may be appropriate when $K \neq 0$, an 
unsupported flexible plate should have its damping modelled in the flexure term; however, our intention in this paper is to show the effects of dissipation. In the present problem we apply hinged-edge conditions at the leading and trailing edges of the plate although there is no restriction on such boundary conditions in the method that follows below.

Assuming the flow to be incompressible and irrotational, a velocity perturbation potential $\phi(x, y, t)$ which satisfies Laplace's equation,

$$
\nabla^{2} \phi=0
$$

is introduced. The unsteady fluid pressure can then be found from the linearised Bernoulli relation

$$
\Delta p=-\rho \frac{\partial \phi}{\partial t}-\rho U_{\infty} \frac{\partial \phi}{\partial x}
$$

where $\rho$ and $U_{\infty}$ are, respectively, the fluid density and flow speed. The plate and fluid motions are coupled through the kinematic boundary condition

$$
\frac{\partial \phi}{\partial y}=\frac{\partial \eta}{\partial t}+U_{\infty} \frac{\partial \eta}{\partial x}
$$

which, in the linearised system, is enforced at $y=0$.

\section{Eigenvalue determination}

(a) A single governing equation for the system

The objective here is to develop a partial solution of the system equations that results in a single differential equation for the system with the interfacial position, $\eta(x, t)$, as the variable.

A suitable solution to Eqn. 2.2, is obtained as the sum of the mean flow plus the effect of a distribution of singularities along the deforming interface that are fundamental solutions to the Laplace equation. In this non-lifting application we choose to use source(-sinks) with strengths denoted $\sigma(x)$. A boundary-element method is then applied. The interface is discretised into a set of $N$ panels, each having constant $\sigma_{i}(i: 1 \rightarrow N)$. The vector of perturbation potential at each panel control point is then given by

$$
\{\phi\}=[\Phi]\{\sigma\},
$$

where $[\Phi]$ is a $(N \times N)$ matrix of influence coefficients with its entries solely determined by the geometry of the discretised interface. Linearisation based on smallamplitude deformations, eliminates the dependence of this matrix on the actual values of $\eta(x, t)$. The no-flux kinematic condition, Eqn. 2.4, is then enforced at the panel control points to yield the required source strengths. The linearised solution is

$$
\{\sigma\}=2 U_{\infty}\left[D_{1}\right]\{\eta\}+2\left[D^{+}\right]\{\dot{\eta}\}
$$

where $\{\eta\}$ is the vector of interfacial displacements at the $N$ evaluation points, the overdot denotes time-differentiation, $\left[D_{1}\right]$ is the first-order spatial differentiation finite-difference matrix operator and $\left[D^{+}\right]$is a matrix operator for the interfacial vertical speed. 
To evaluate the pressure perturbation, the discretised form of Eqn. 2.3 is used, evaluating the tangential velocity perturbation using Eqns. 3.1 and 3.2, together with the direct substitution of the perturbation potential to give

$$
\begin{aligned}
-\{\Delta p\} & =2 \rho U_{\infty}^{2}[T]\left[D_{1}\right]\{\eta\}+2 \rho U_{\infty}[T]\left[D^{+}\right]\{\dot{\eta}\} \\
& +2 \rho U_{\infty}[T][\Phi]\{\dot{\eta}\}+2 \rho[\Phi]\left[D^{+}\right]\{\ddot{\eta}\}
\end{aligned}
$$

where $[T]$ is the matrix of tangential-velocity influence coefficients. The form of Eqn. 3.3 shows the pressure to comprise the hydrodynamic stiffness (curvature effects), followed by two terms that yield the hydrodynamic damping (Coriolis' effects) and the final term that represents the hydrodynamic inertia (added-mass effects). The foregoing solution method for the flow field is described in more detail in Lucey et al. (1997) wherein expressions for the various influence coefficients are listed.

We now proceed to assemble the system equation. The wall equation is written in finite-difference form using a set of $N$ lumped-mass points that corresponds to the boundary-element panel control points, hence

$$
\rho_{m} h[I]\{\ddot{\eta}\}+d[I]\{\dot{\eta}\}+\left(B\left[D_{4}\right]+K[I]\right)\{\eta\}=-\{\Delta p\},
$$

where $[I]$ is the identity matrix and $\left[D_{4}\right]$ is the fourth-order spatial differentiation (penta-diagonal) matrix operator. Finally, the pressure-perturbation vector of Eqn. 3.3 is substituted into Eqn. 3.4 and the result re-arranged to give

$$
\{\ddot{\eta}\}=[E]\{\dot{\eta}\}+[F]\{\eta\}
$$

where

$$
\begin{aligned}
& {[E]=\left(\rho_{m} h[I]-2 \rho[\Phi]\left[D^{+}\right]\right)^{-1}\left(2 \rho U_{\infty}[T]\left[D^{+}\right]+2 \rho U_{\infty}[T][\Phi]-d[I]\right)} \\
& {[F]=\left(\rho_{m} h[I]+2 \rho[\Phi]\left[D^{+}\right]\right)^{-1}\left(2 \rho U_{\infty}^{2}[T]\left[D_{1}\right]-B\left[D_{4}\right][\Phi]-K[I]\right)}
\end{aligned}
$$

(b) State-space solution

We now solve Eqn. 3.5 using a standard state-space method. The second order $N \times N$ system is transformed to the following first-order $2 N \times 2 N$ system

$$
\{\dot{w}\}=[H]\{w\},
$$

where

$$
[H]\{w\}=\left[\begin{array}{cc}
0 & I \\
-F & E
\end{array}\right]\left\{\begin{array}{l}
\{\eta\} \\
\{\dot{\eta}\}
\end{array}\right\},
$$

for the new variable $w$. Assuming that all parts of the system move with the complex frequency, $s=s_{R}+$ is $s_{I}$, we can write

$$
w=W \exp (s t)
$$

and substituting this into Eqn. 3.6, yields

$$
(s[I]-[H])\{W\}=0,
$$

and the solution of $\operatorname{det}(s[I]-[H])=0$ then generates the eigenvalues. These have been evaluated using the ARPACK solver through the EIGS command in the MATLAB software. Having found the eigenvalues, these can then, in turn, be substituted 
back into Eqn. 3.9 to extract the complex eigenmode, $\{W\}^{T}$ for the $N$ interfacial points. In continuous space we can therefore write $W(x)=W_{R}(x)+\mathrm{i} W_{I}(x)$. Thus, using Eqn. 3.8, the motion of the plate in each eigenmode can be written in the form

$$
\Re[\eta(x, t)]=\exp \left(s_{R} t\right)\left(W_{R}(x) \cos \left(s_{I} t\right)+W_{I}(x) \sin \left(s_{I} t\right)\right) \quad .
$$

This motion will be plotted in the results presented below.

\section{(c) Galerkin solution}

The formulation above can, alternatively, be used to construct a traditional Galerkin solution for the flow-structure system. We present this here because in generating the results discussed below we have conducted comparisons of the eigenmodes determined by the direct state-space determination and those predicted by the Galerkin method based upon a finite collection of in-vacuo orthogonal modes.

Using Eqn. 1.1, a trial solution based upon an assembly $R$ in-vacuo orthogonal modes is proposed, hence

$$
\{\eta\}=[S]\{A\} \exp (s t)
$$

where $[S]$ is an $N \times R$ matrix that comprises the modal deflections at the $N$ interfacial points of each of the $R$ modes $(\sin (n \pi x / L)$ for $n: 1 \rightarrow R$ in this study) and $[A]$ is a vector of length $R$ containing the complex modal intensities. Upon substitution of Eqn. 3.11 into Eqn. 3.5 and subsequent multiplication by $[S]^{T}$, the following $R \times R$ matrix equation is developed,

$$
\left(s^{2}[S]^{T}[S]-s[S]^{T}[E][S]-[S]^{T}[F][S]\right)\{A\}=0 .
$$

We remark that the generalised hydrodynamic integrals for inertia, damping and stiffness that feature in the Galerkin method are effectively evaluated in the respective matrix products $[S]^{T}[S],[S]^{T}[E][S]$ and $[S]^{T}[F][S]$. Equation 3.12 is solved using conventional methods for the complex frequencies, $s$, and then the relative modal intensities, $\{A\}^{T}$ are calculated. Finally, the form of the motion can be assembled as the weighted sum of the $R$ in-vacuo modes selected to represent the panel dynamics.

\section{Results}

(a) Simple elastic plate

Prior to presenting results for a fluid-loaded flexible plate, we briefly provide evidence of the integrity and accuracy of the numerical procedure using in vacuo oscillations. The state-space method has been used with $\rho=0$ to generate the first four in-vacuo modes. Using $N=200$ for the spatial discretisation, excellent agreement with the theoretical eigenmodes, $\sin (n \pi x / L)$ for $n=1,2,3,4$, is found with the frequencies lying within $0.5 \%$ of the theoretical values generated by

$$
s_{I}=\left(\frac{n \pi}{L}\right)^{2} \sqrt{\frac{B}{\rho_{m} h}} .
$$

Article submitted to Royal Society 
In the results of this section, we use the non-dimensional scheme of Lucey et al. (1997) that is appropriate to the finite system studied herein. Thus the nondimensional control parameter (or stiffness ratio), vertical displacement and time are given by

$$
\Lambda^{F}=\frac{\rho U_{\infty}^{2} L^{3}}{B}, \quad \eta^{\prime}=\left(\frac{1}{h}\right) \eta, t^{\prime}=\left\{\frac{\sqrt{B / \rho_{m} h}}{L}\right\}\left(\frac{1}{h}\right) t
$$

The term in braces in Eqn. 4.2c above can be interpreted as the lower limit of the free-wave phase speed for the finite wall structure; Eqn. $4.2 \mathrm{c}$ is also used to non-dimensionalise the radian frequency in the results of this paper.

Figures $2 \mathrm{a}$ and $2 \mathrm{~b}$ explore the dependence of the system eigenvalues upon the numerical discretisation used in the present method while Figures $2 \mathrm{c}$ and $2 \mathrm{~d}$ compares the converged results of the present method with those obtained using a Galerkin solution. Each figure shows the variation of eigenvalues with $\Lambda^{F}$ that can be interpreted as a measure of flow speed for given plate properties; Figures 2a and $2 \mathrm{c}$ depict the oscillatory, $s_{I}^{\prime}$, part of the eigenvalues while Figures $2 \mathrm{~b}$ and $2 \mathrm{~d}$ show the corresponding growth/decay, $s_{R}^{\prime}$, parts of the eigenvalues. In order to discern the effects of discretisation we have restricted the range of the vertical axes in each plot. In Figures $2 \mathrm{a}$ and $2 \mathrm{~b}$ convergence of the present method is clearly evident as the number of collocation points, $N$, is increased; note that the lines for $N=100$ and $N=200$ are almost coincident. Figures 2c and 2d demonstrate that the Galerkin method yields more rapid, spectral, convergence than the present method. Comparing these and the $N=200$ result of the present method indicates agreement for the divergence-onset flow speed and the divergence branches as $\Lambda^{F}$ is increased. Beyond this range of $\Lambda^{F}$, where higher-order modes increasingly contribute to the system eigenstate, there is a small but perceptible difference between the predictions of the two methods although the solution morphology is the same for both methods. However, the focus of this paper is upon linear-instability onset and its mechanisms in the low-value range of $\Lambda^{F}$ where good agreement is found between the results of the present method and those due to the Galerkin method.

In Figure 3 we re-plot the results of Figure $2 \mathrm{a}$ and $2 \mathrm{~b}$ for the case $N=200$ restricting the range of both axes so as to focus on the lowest two flow-structure eigenmodes that are the first to be destabilised with increasing $\Lambda^{F}$. Of course, all of the higher-frequency modes are retained in the calculation and the neutral stability of these modes, throughout the flow-speed range presented, appears as the solid line $s_{I}^{\prime}=0$ in Figure 3b. Four zones of response are noted in Figure 3. At low, pre-divergence, flow speeds neutrally-stable oscillatory motion prevails. Thereafter, divergence sets in; Mode 1 bifurcates to give a pair of $\pm s_{R}^{\prime}$ non-oscillatory solutions while Mode 2 continues to be neutrally stable. At higher $\Lambda^{F}$, there is a neutrally-stable region of divergence recovery wherein Mode 1 once again becomes oscillatory. Finally, Modes 1 and 2 coalesce to give a pair of solutions, $\pm s_{R}^{\prime}+\mathrm{i} s_{I}^{\prime}$, with non-zero oscillatory part; the unstable behavior associated with $+s_{R}^{\prime}$ in this region is commonly known as flutter. This sequence of broad changes is very well known, corresponding plots having been presented by, for example, Weaver \& Unny (1971), Garrad \& Carpenter (1982) and Lucey \& Carpenter (1993) although these predictions were based on very limited number of modes being included in the calculation. While, the present, more precise, calculations confirm these general 

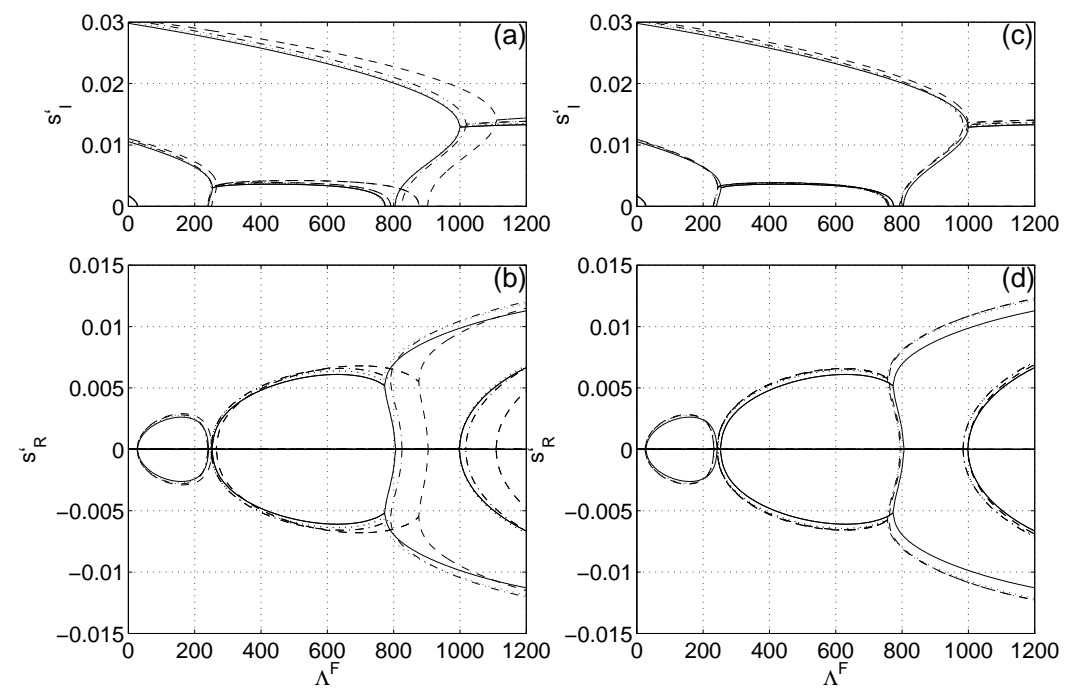

Figure 2. The effect of discretisation on the prediction of the variation of system eigenvalues with flow-to-wall stiffness ratio (applied flow speed for a plate of given properties), (a) and (c) depict the imaginary (oscillatory) part, and (b) and (d) depict the real (positive = growth, negative $=$ decay) part. Present direct-method predictions in (a) and (b), for: - ,$--- N=20 ;-\cdot-, N=50 ; \cdots, N=100 ;-, N=200$. Galerkin-method predictions in (c) and (d), for first: - - - - , 5 modes; - - -, 10 modes; $\cdots, 20$ modes; and where is the direct result for $N=200$.

predictions, below we investigate in rather more detail the system behaviour and how it is affected by changes from the model of a standard homogeneous elastic plate held at just its ends.

We first illustrate the motion of the standard plate in the four different zones of response. In the low-speed pre-divergence zone, the neutrally-stable oscillatory motion of the first three eigenmodes is shown in Figures 4a-c, at zero flow speed $\left(\Lambda^{F}=0\right)$, and Figures $4 \mathrm{~d}-\mathrm{f}$, at $\Lambda^{F}=17.6$. In these, and following depictions of eigenmodes, deformations, as determined by Eqn. 3.10, are normalised to the final deflection arising from a sequence of time steps; this final deformation is indicated by the use of a thick line. At zero flow speed only the added mass of the fluid is present in the loading. Accordingly, the motion is symmetrical about the panel's mid-point. While Modes 1 and 2 are very close in shape to the first two in-vacuo sinusoidal modes, the shape of Mode 3 has clearly been affected by the distribution of fluid inertia. For a non-zero pre-divergence flow speed, close inspection of Figures 4d-f reveals that each of the plate deflections, for all three modes, do not have mirrorimage symmetry about the line $x / L=0.5$. Moreover, exact standing-wave nodes no longer exist for Modes 2 and 3; these become quasi-nodes, the importance of which is discussed in sub-section (e) below. Animations reveal that these features give the visual effect of wave travel superimposed upon the oscillatory standing wave; as the amplitude decreases through the cycle, upstream travel is perceived whilst as it increases, downstream travel is seen.

In the divergence range of flow speeds the eigenvalue for Mode 1 is purely real, comprising a $\pm s_{R}^{\prime}$ pair of solutions, the positive branch indicating instability. Fig- 

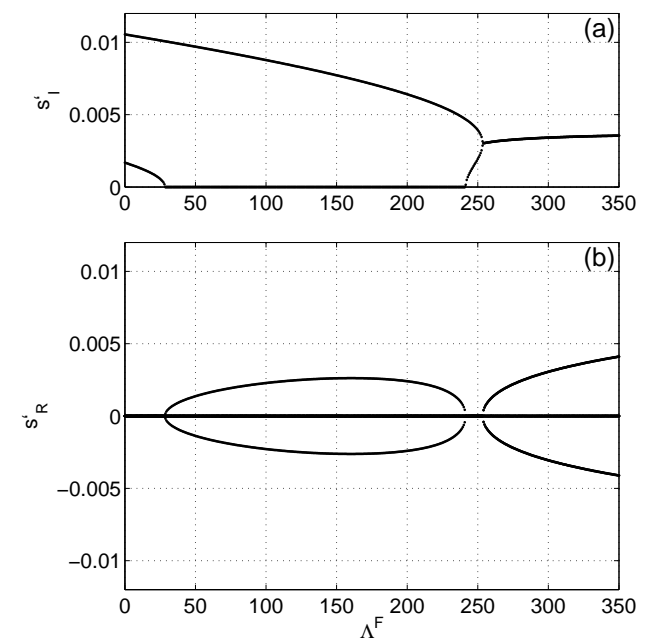

Figure 3. Elastic-plate. Variation of the two lowest eigenvalues with flow-to-wall stiffness ratio (applied flow speed for a plate of given properties). (a) imaginary (oscillatory) part, (b) real (positive $=$ growth, negative $=$ decay) part.

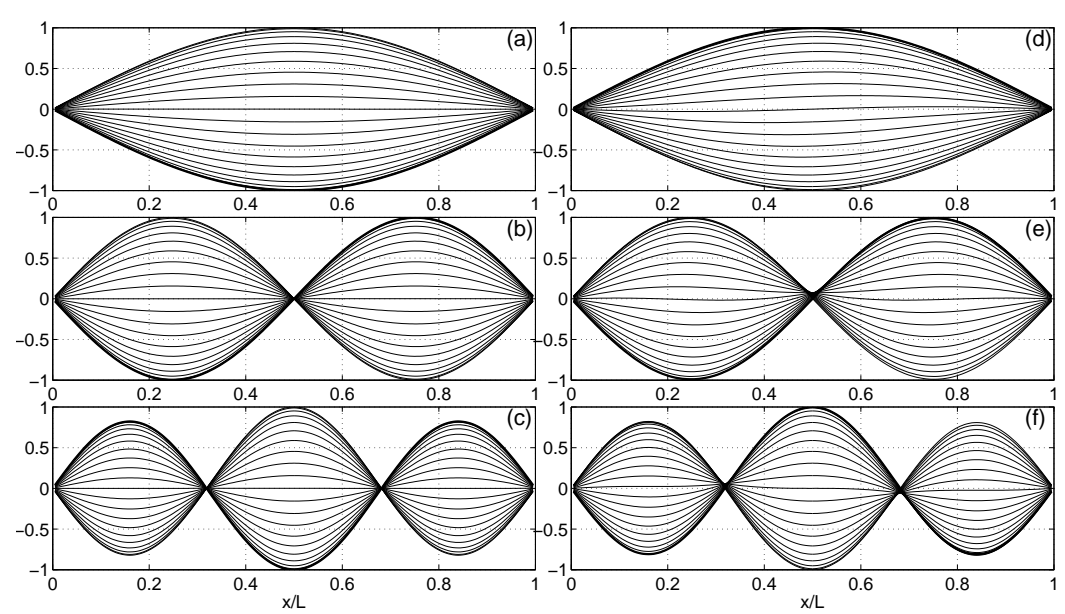

Figure 4. Elastic plate: snapshots of the wall motion over one half-cycle of oscillation in the pre-divergence range of flow speeds for $\Lambda^{F}=0$, (a) Mode 1, (b) Mode 2, and (c) Mode 3; and at $\Lambda^{F}=17.6$, (d) Mode 1, (e) Mode 2, and (f) Mode 3. The thick line indicates the final wall position in the time-sequence of plots.

ures 5a-c show the unstable amplifying solution for a series of increasing flow speeds, $\Lambda^{F}=70.5,138$ and 228 , all lying within the divergence zone while Figures 5 d-f show the companion decaying solutions for the same flow speeds. Clearly, it is the amplifying mode that will dominate the wall deformation with the passage of time. The shape of the unstable mode is strongly dependent upon the flow speed, being very close to the fundamental mode exactly at the divergence-onset speed but increasingly evolving towards a Mode- 2 shape as $\Lambda^{F}$ is increased. The same is noted for 


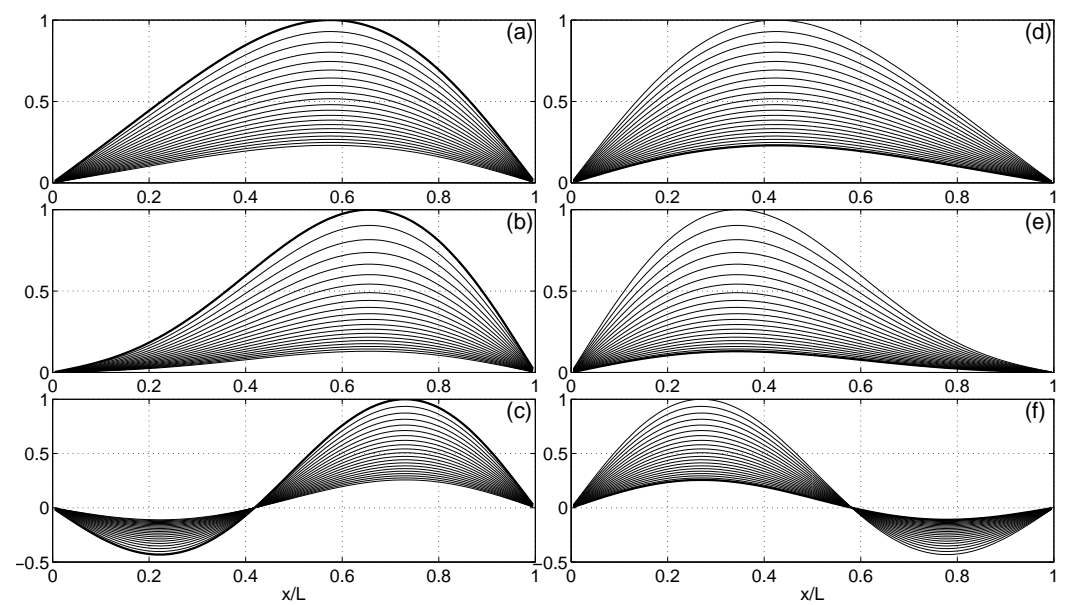

Figure 5. Elastic plate. Snapshots of wall motion in the divergence range of flow speeds: of Mode-1 amplifying for (a) $\Lambda^{F}=70.5$, (b) $\Lambda^{F}=138$, and (c) $\Lambda^{F}=228$; and Mode-1 attenuating for (d) $\Lambda^{F}=70.5$, (e) $\Lambda^{F}=138$, and (f) $\Lambda^{F}=228$, for the period $t^{\prime}: 0 \rightarrow 793$ over 20 time steps each of duration $\Delta t^{\prime}=39.6$ The thick line indicates the final wall position in the time-sequence of plots.

the decaying mode although its apparent distortion is in the opposite, upstream, direction. We may then speculate that in an initial-value problem, the transient motion of the panel, as a combination of many modes, would appear as a mixture of downstream-directed amplifying motion and upstream-directed attenuating motion. Also present would be various higher-frequency neutrally-stable modes. This combination of downstream distortion during amplification and upstream distortion during attenuation resembles that seen in the non-linear study of panel flutter of Lucey et al. (1997) wherein induced tension both restricted the amplification and drove the attenuation in limit-cycle motions. What can be concluded here is that, even in the linear regime of divergence, travelling-wave motions would be discerned in a real situation.

The third type of behavior is found in the divergence-recovery zone wherein Mode 1 comprises neutrally-stable low-frequency oscillatory motion as predicted by Figure 3. Figure 6 , at $\Lambda^{F}=254$, shows the shapes of both Mode 1 and Mode 2, the key feature being their close similarity in the zone prior to their coalescence at a higher flow speed. In the results of the next sub-section, we will find that this unusual zone of neutrally stable behavior between divergence and flutter instabilities would not exist for a plate with any level of structural damping.

The final type of behavior arising from the eigenvalues of Figure 3 is that of flutter caused by the coalescence of Modes 1 and 2 . The unstable mode, at $\Lambda^{F}=281$ is plotted in Figure 7a having suppressed the amplification so as to highlight the mode shape. This figure, together with animations, reveal that the visual perception of this instability is that of a downstream-travelling amplifying wave. In contrast, Figure $7 \mathrm{~b}$ results from the attenuating branch at the same $\Lambda^{F}$ with the decay suppressed. This mode clearly contains a strong component of the third mode that becomes a significant part of the low-frequency system response at these higher flow speeds. 


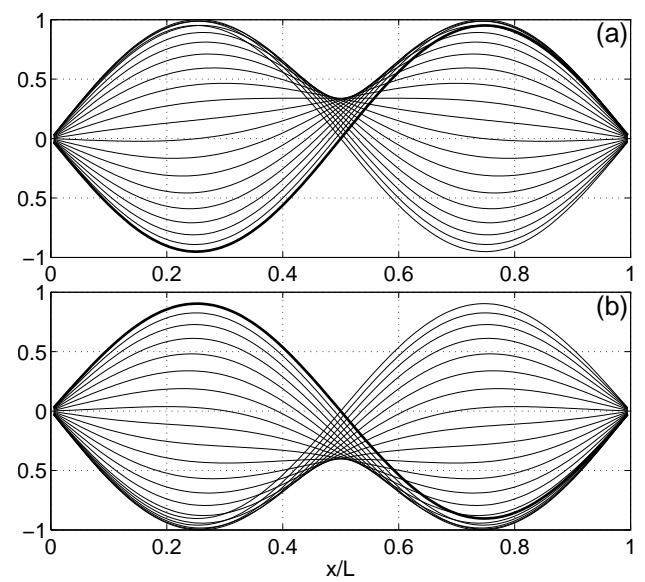

Figure 6. Elastic plate. Snapshots of wall motion over one half-cycle of oscillation in the divergence-recovery zone of flow speeds at $\Lambda^{F}=254$, in (a) Mode 1, and (b) Mode 2. The thick line indicates the final wall position in the time-sequence of plots.

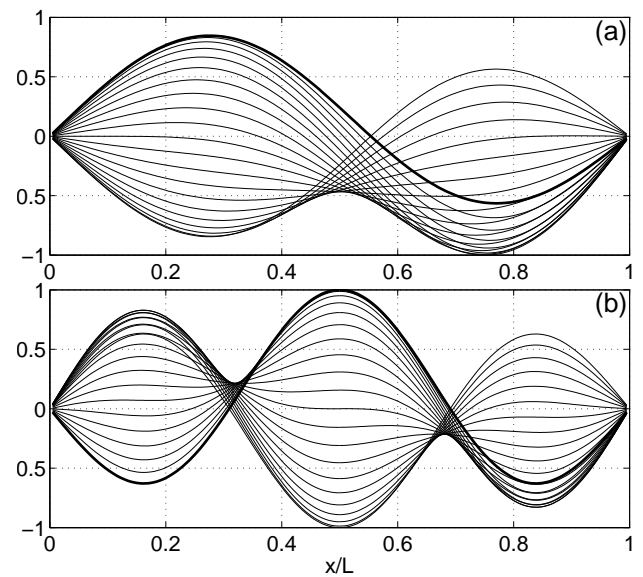

Figure 7. Elastic plate. Mode shapes for $\Lambda^{F}=281$ lying in the modal-coalescence flutter instability range of flow speeds, for one half-cycle of oscillation, (a) lowest-frequency mode, and (b) second mode. Note that the growth/attenuation has been suppressed so as to highlight the mode shapes. The thick line indicates the final wall position in the time-sequence of plots.

\section{(b) Flexible plate with structural damping}

We now consider the effect of energy dissipation within the flexible plate. A non-dimensional damping coefficient,

$$
d^{\prime}=\left(\frac{L^{2}}{2 \sqrt{\rho_{m}\left(B \pi^{4}+K L^{4}\right)}}\right) d
$$



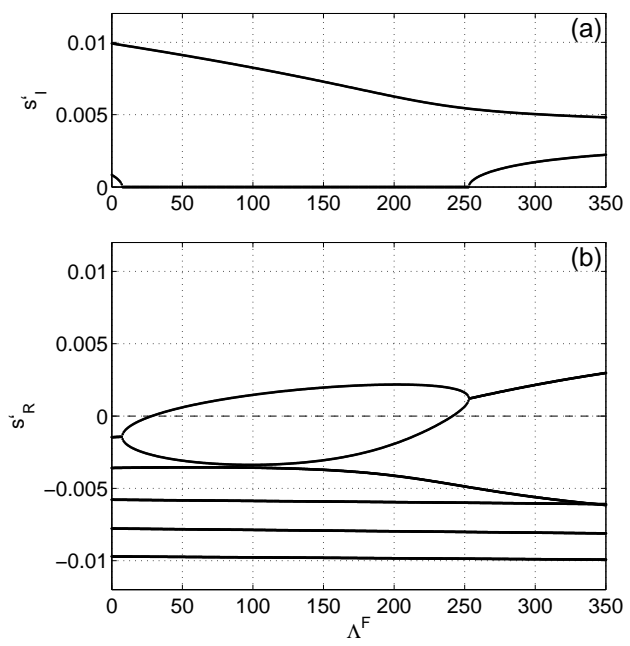

Figure 8. Flexible plate with structural damping set at $d^{\prime}=6.13$ : variation of the two lowest eigenvalues (five lowest for the real parts) with flow-to-wall stiffness ratio (applied flow speed for given plate properties). (a) imaginary (oscillatory) part, (b) real (positive = growth, negative $=$ decay) part.

with a value of 6.13 is introduced herein (with $K=0$ ) for illustrative purposes. This relatively high value would yield a $50 \%$ amplitude reduction over one cycle of oscillation for the fundamental in-vacuo mode of the flexible plate.

Figure 8 shows the variation of the system eigenvalues with $\Lambda^{F}$ and can be compared with the results of Figure 3 for an undamped plate. Note that in Figure $8 \mathrm{~b}, s_{R}^{\prime}$ is plotted for each of Modes 1-5 whereas only Modes 1 and 2 oscillatory branches are within scale in Figure 8a. While there continues to be a low-speed zone of oscillatory behavior, there is now a new zone of non-oscillatory damped behavior of Mode 1 before the divergence-onset flow speed is reached. The actual divergenceonset flow speed is unchanged by the addition of damping; this is because the instability is static exactly at onset. The mode shapes in the new zone of behavior are plotted in Figure 9. It is evident that transient motion in response to some form of initial excitation could only feature oscillations of the second and higher modes. Within the divergence zone of flow speeds, the plate behavior is qualitatively the same as for the undamped plate except that the instability growth rate is reduced by the structural damping.

A second key difference between damped and undamped plate dynamics occurs in the post-divergence zone. Figure 8 shows that, with increasing $\Lambda^{F}$, the divergence instability merges into flutter instability. There is now neither a divergence-recovery zone nor an explosive onset of flutter through exact modal coalescence. Results for different values of damping reveal that the solution morphology seen in Figure 8 is unchanged. Thus, even for an incremental level of damping, there is no divergencerecovery zone and unstable behavior is found at all flow speeds above that at which divergence first occurs. Thus, for any real panel, in which some level of structural energy dissipation must be present, divergence and flutter would not be seen as a distinct events. 


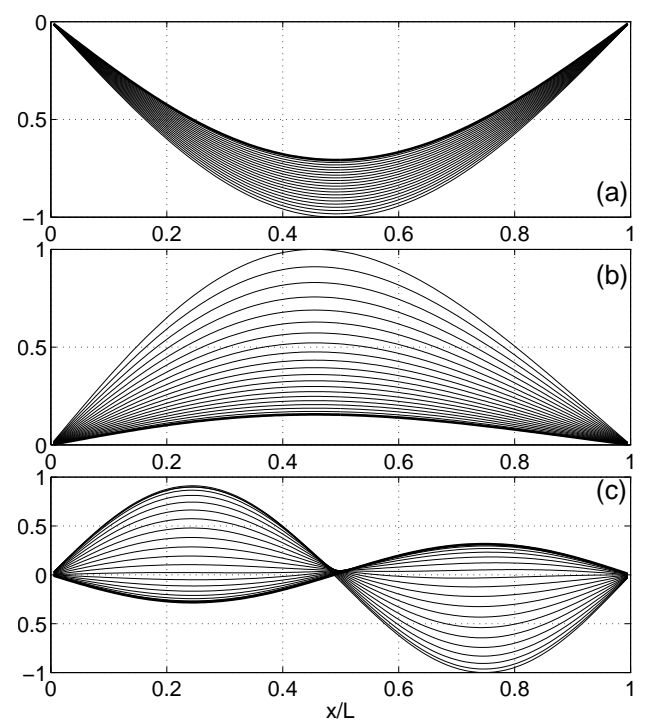

Figure 9. Elastic plate with structural damping: snapshots of the wall motion in the pre-divergence range of flow speeds with $\Lambda^{F}=20$, (a) Mode 1 upper branch, (b) Mode 1 lower branch, for $t^{\prime}: 0 \rightarrow 793$ over 20 time steps each of duration $\Delta t^{\prime}=39.6$ and (c) Mode 2 (over one half-cycle of oscillation). The thick line indicates the final wall position in the time-sequence of plots.

\section{(c) Spring-backed Flexible plate}

We now present illustrative results for a flexible panel that possesses two types of structural force. The combination of flexural rigidity and foundation-spring stiffness means that hydro-elastic instability is governed by higher-order modes. Accordingly, travelling-wave, or local, theoretical methods, using Eqn. (1.2) as the deflection form, are most often used in this type of system; however, these neglect the effect of the panel's end restraints.

For this sub-section, we use a non-dimensional flow-structure stiffness ratio defined as

$$
\Lambda^{I}=\frac{3 \pi \rho U_{\infty}^{2}}{\left(3 B K^{3}\right)^{\frac{1}{4}}},
$$

based upon the length scale of the critical hydroelastic mode as opposed to the length of the entire flexible wall, $L$; see Lucey et al. (1997). Again this parameter may be considered as a measure of flow speed for given flexible-wall properties.

Figure 10 displays the variation of the first 40 system eigenmodes with $\Lambda^{I}$ for a damped wall that has $d^{\prime}=0.56$. It can be seen, from the plot of the real part in Figure 10b, that the lowest $\Lambda^{I}$ at which an amplifying response occurs is at approximately 128 where one branch of $s_{R}$ first crosses into the positive quadrant. However, it can be seen this critical mode marks the edge of a response-envelope that is dense with unstable modes. Thus, the flexible wall's response throughout the flow-speed range is characterised by fairly complex modes and combinations thereof. Figures 11a-c show the three modes closest to the null eigenvalue at $\Lambda^{I}=123$ in the pre-instability range of flow speed. Clearly, the motion of the flexible wall is 

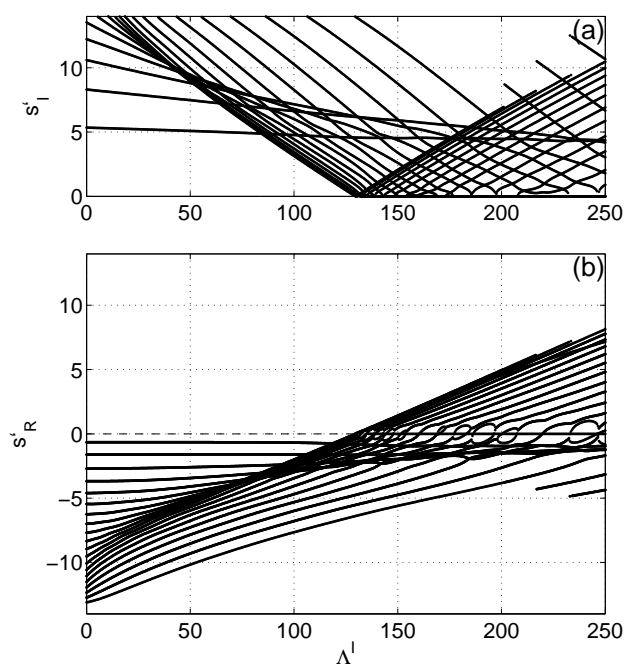

Figure 10. Spring-backed flexible plate with structural damping $\left(d^{\prime}=0.56\right)$ : variation of the 40 eigenvalues closest to $0+0 \mathrm{i}$ with flow-to-wall stiffness ratio (applied flow speed for given plate properties). (a) imaginary (oscillatory) part, (b) real (positive = growth, negative $=$ decay) part.

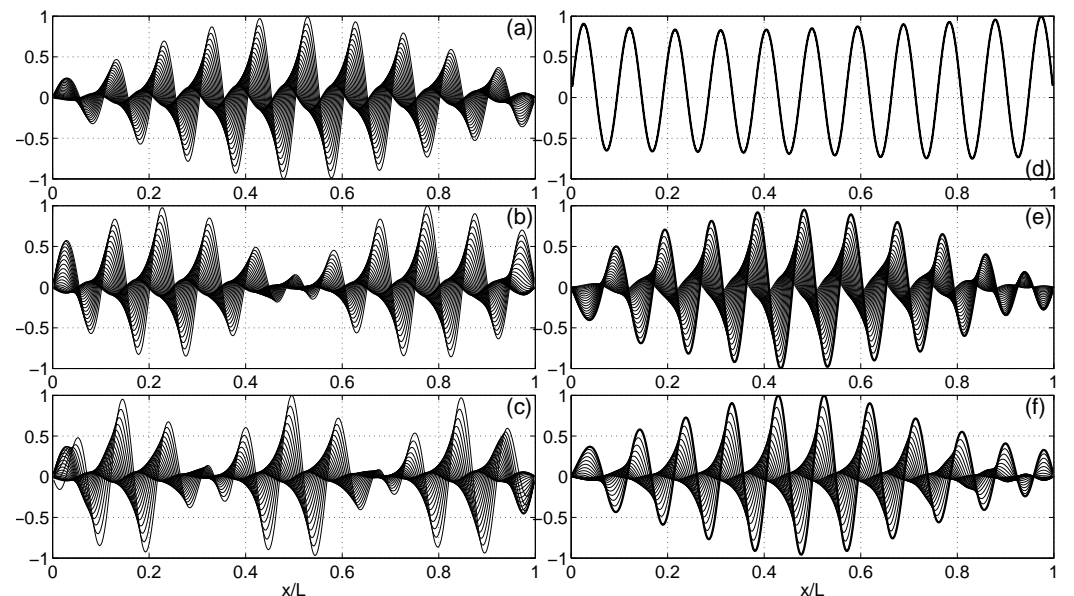

Figure 11. Spring-backed flexible plate with structural damping $\left(d^{\prime}=0.56\right)$ : snapshots of wall motion at subcritical (pre-divergence) $\Lambda^{I}=123$ for (a)-(c) the three modes closest to $0+0 \mathrm{i}$ in order of increasing frequency; and for the most unstable mode at post-critical flow speeds, (d) $\Lambda^{I}=130$, (e) $\Lambda^{I}=136$, and (f) $\Lambda^{I}=140$. All for the period $t^{\prime}: 0 \rightarrow 4.17$ over 20 time steps each of duration $\Delta t^{\prime}=0.21$. The thick line indicates the final wall position in the time-sequence of plots.

dominated by upstream-travelling waves as opposed to oscillatory standing waves. Figures 11d-f, respectively show the most unstable eigenmodes for each of $\Lambda^{I}=$ 130,136 and 140. Downstream-travelling wave motion is seen to dominate most locations of the flexible wall although standing-wave flutter is evident in the regions 
adjacent to the leading and trailing edge of the panel where wave travel is most inhibited by the plate's fixed-end conditions. Thus, for wall structures that are more complex than a simple panel, there is no evidence of a global static standing-wave type of divergence instability. The present results also indicate that a travellingwave type of analysis becomes an appropriate approximation for interior regions of the flexible wall although it would fail to capture the spatial modulation of the global response seen, for example, in Figures 11e and 11f.

\section{(d) Inhomogeneous flexible plates and complex boundary conditions}

We present here two examples of unsupported flexible elastic plates for which the extraction of eigenmodes using the present method is particularly suited. In the first, we apply the present method to a wall that has spatially varying flexural rigidity of the form $B(x)=\bar{B}[1+(2 x / L-1) \lambda]$ in Eqn. 2.1 and non-dimensionalise the results using the mean value of flexural rigidity, $\bar{B}$. This allows comparison with our earlier results in Figure 3 for a plate of homogeneous properties. Figures 12a and $12 \mathrm{~b}$ display the variation of the eigenvalues with $\Lambda^{F}$ for $\lambda=-0.95$ (stiffest near the leading edge) while Figures $12 \mathrm{c}$ and $12 \mathrm{~d}$ display the corresponding results for $\lambda=0.95$ (stiffest near the trailing edge). The frequency ranges plotted in Figure 12 have again been chosen to highlight the behaviour of the two lowest modes although, as before, all eigenmodes are evaluated. Clearly, the spatial variation of flexural rigidity has a marked effect on system stability. In both cases the divergence-onset value of $\Lambda^{F}$ is higher than that of a homogeneous plate. For $\lambda=-0.95$ all modes in the pre-divergence range of $\Lambda^{F}$ are now damped. The unstable mode shapes in the divergence loop remain similar to those seen in Figures $5 \mathrm{a}-\mathrm{c}$ but a reduced growth rate is evident and there is amplification bias towards the downstream half of the plate that is less stiff. A further change occurs in the transition from divergence to flutter as $\Lambda^{F}$ is increased. There is now clearly a range of $\Lambda^{F}$ for which $s_{I}^{\prime}$ is negative for all modes indicating damped behaviour of the flow-structure system. Thereafter, the exact coupling of Modes 1 and 2 seen in Figure 3 does not occur and the severity of the flutter instability is reduced. The overall effect on the system is very much as if it were damped. In contrast, Figures $12 \mathrm{c}$ and $\mathrm{d}$ show that for $\lambda=0.95$ the reverse occurs and the system behaves as if it were negatively damped. Thus, of particular importance is the existence of pre-divergence instability that is most pronounced in a single-mode flutter amplification of Mode 1 . While the inclusion of some structural damping in the plate could eliminate this instability, its existence is of interest and its causes are explored in sub-section (e) below.

Figure 13 shows eigenvalue and eigenmode results for a flexible plate with constant $B$ but with an additional interior boundary condition. At $x / L=0.3$ we stipulate that the plate is constrained by a hinge placed in the plane of the undeformed flexible plate; thus, the conditions of zero plate displacement and bending moment are enforced at this point. The divergence behaviour is now dominated by the longest extent of flexible plate between restraints. This is clearly seen by comparing the divergence mode shapes in the region $x / L: 0.3 \rightarrow 1$ in Figures 13c-e with those over the full expanse of the flexible wall for the standard case in Figures 5a-c. The eigenvalue plots of Figures $13 \mathrm{a}$ and $13 \mathrm{~b}$ indicate that the added restraint has increased the divergence-onset value of $\Lambda^{F}$. This is to be expected because of the effective reduction in the length of the divergence-prone part of the 

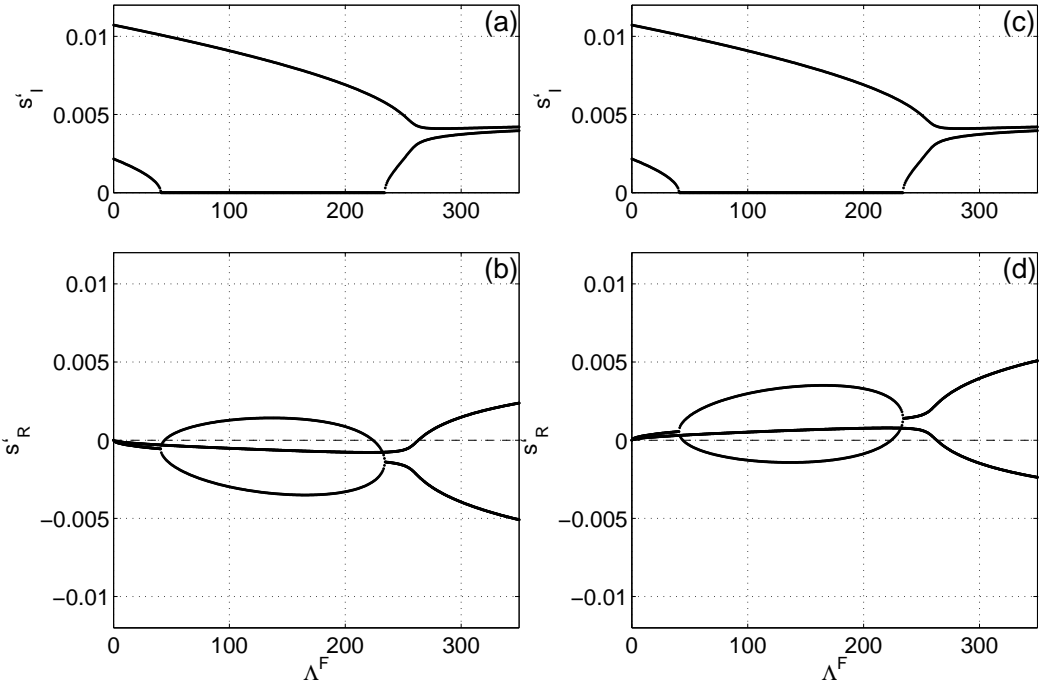

Figure 12. Effect of material inhomogeneity on the variation of the two lowest eigenvalues with flow-to-wall stiffness ratio (applied flow speed for a plate of given properties), (a) and (c) depict the imaginary (oscillatory) part, and (b) and (d) depict the real (positive = growth, negative = decay) part. Decreasing, (a) and (b), and increasing, (c) and (d), flexural rigidity from plate leading edge, with a $95 \%$ variation about the value at $x / L=0.5$ in each case.

flexible plate to the region $x / L: 0.3 \rightarrow 1$. However, we also remark that if we non-dimensionalise $\Lambda^{F}$ using $0.7 L$ instead of $L$, the divergence-onset flow speed is marginally higher than that of a simple wall of length $0.7 L$; this suggests that the wall motions upstream of $x / L=0.3$ in the present configuration have a stabilising effect with respect to divergence-onset. However, these motions also exercise a more subtle effect in that the $s_{R}^{\prime}$-divergence loop is distorted into the positive quadrant thereby suggesting a more severe instability. Furthermore, a divergence-recovery zone is now absent. Flutter evolves smoothly from divergence instability in much the same way that was effected by structural damping as seen in Figure 8. We therefore note that the introduction of a singular spatial homogeneity modifies the phase relationships between structural and fluid forces and thereby alters the stability characteristics of the flexible plate. This assertion is borne out by one further new feature of the eigenvalue plots. One of the neutrally stable branches at very low speeds in the standard case of Figure 3 is now unstable, even to vanishingly small values of $\Lambda^{F}$. We find that this is single-mode flutter of Mode 3 that is similar in shape to the third in-vacuo mode. The identity of this mode is perhaps unsurprising because the added constraint is placed at $x / L=0.3$. If we place the constraint at $x / L=0.25$ we then find that the third mode is stable whereas the fourth mode succumbs to single-mode flutter. This type of flutter instability would be attenuated, or even eliminated, by the action of structural damping in the plate. This type of phenomenon has been found in related flow-structure systems; Païdoussis (1998) shows that the addition of an end restraint to a fluid-conveying cantilevered flexible pipe can precipitate fluttering motions of the pipe. However, this is the 

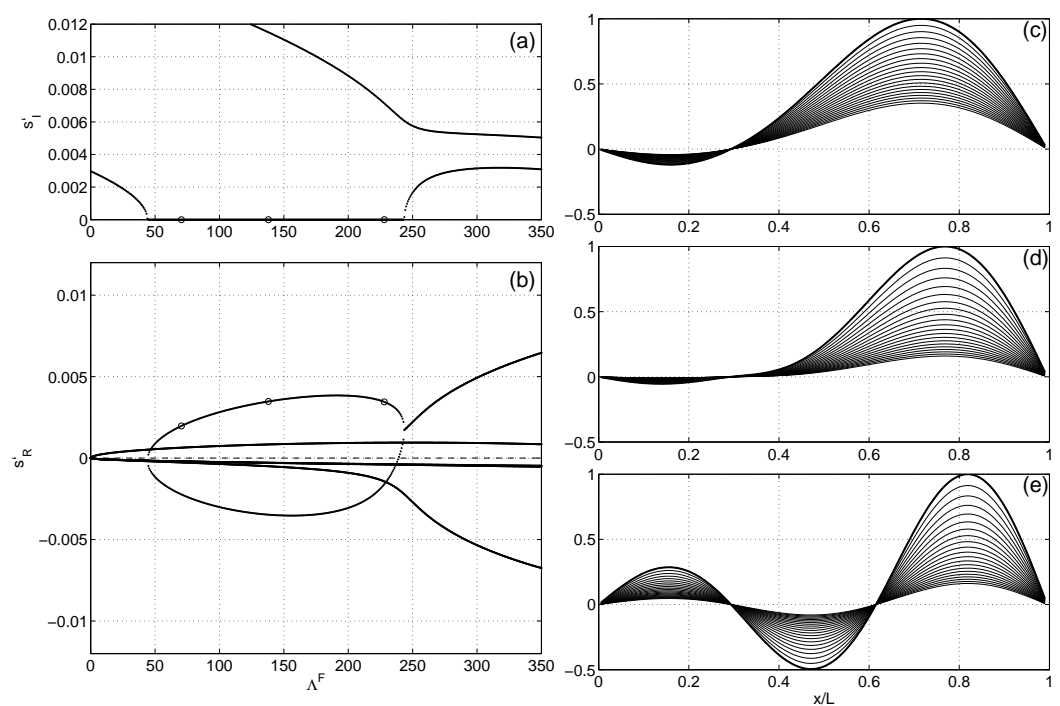

Figure 13. Elastic plate with a hinged boundary constraint at $30 \%$ chord. Variation of the two lowest eigenvalues (five lowest for the real parts) with flow-to-wall stiffness ratio (applied flow speed for a plate of given properties). (a) imaginary (oscillatory) part, (b) real (positive $=$ growth, negative $=$ decay) part. Snapshots of wall motion in the divergence range of flow speeds, Mode-1 amplifying, for $t^{\prime}: 0 \rightarrow 793$ over 20 time steps each of duration $\Delta t^{\prime}=39.6$ at: (c) $\Lambda^{F}=70.5$, (d) $\Lambda^{F}=138$, and (e) $\Lambda^{F}=228$. In (c)-(e) the thick line indicates the final wall position in the time-sequence of plots.

first prediction of it occurring for the open flow over a flat flexible plate; below, we identify the underpinning mechanism of this type of pre-divergence instability.

\section{(e) Mechanism for single-mode flutter at pre-divergence flow speeds}

We now show why the introduction of spatial inhomogeneity, either through varying material properties or the introduction of an additional restraint, can lead to a further type of instability, single-mode flutter, existing in the flow-structure system. During the plate's motion the spatial distribution of work done by the fluid over one cycle of oscillation, $W(x)$, is the time integral of $(-\Delta p) \dot{\eta}$ over one period of oscillation. Spatial integration of $W(x)$ over the entire panel then yields the total work done at the interface over one cycle. For an elastic plate, if this total is positive, then the plate's motion amplifies, if zero it is neutrally stable and if negative it is effectively damped. When structural damping is included it is the sign of the difference between this energy transfer and dissipation that determines stability. Without performing such integrations, it is clear that a phase-angle difference between pressure and plate velocity of $n \pi / 2$ gives $W(x)=0$ when $n$ is an odd integer (orthogonal), a positive value when $n / 2$ is zero or even (in-phase), and a negative value when $n / 2$ is odd (anti-phase). A departure, $\pm \psi$, from any of these cases also provides a mechanism for irreversible energy transfer between fluid and structure.

Figure 14 shows the effect of spatially varying flexural rigidity on the phaseangle difference between pressure and plate velocity, or relative phase, for Mode 1 at the pre-divergence value $\Lambda^{F}=25$ for the two cases, $\lambda=-0.95$ and 0.95 
presented in Figure 12, and for $\lambda=0$, the homogeneous-plate case of Figure 3. In addition to plotting the relative phase for the total pressure that determines the energy transfer, we also plot those of its constituent parts, the hydrodynamic stiffness and damping identified in Eqn. 3.3, that contribute to energy flux at the fluid-structure interface; see Crighton \& Oswell (1991) and Lucey et al. (2003) for a detailed discussion. Focusing first on the homogeneous case $\lambda=0$ in Figure 14b, for which the mode is neutrally stable, it is seen that exact orthogonality of the total pressure is only found at $x / L=0.5$. Departures, $\pm \psi$, from this occur in both upstream and downstream halves of the plate and thus localised energy transfer occurs. However, these transfers must sum to zero because of the antisymmetry about the point of exact orthogonality at $x / L=0.5$ and thus the mode is neutrally stable. While the corresponding results for $\lambda=-0.95$ and $\lambda=0.95$ in Figures 14a and 14b respectively also evidence the same antisymmetry, $x / L=0.5$ is not a point of exact orthogonality and thus a net energy transfer from(to) the plate occurs leading to the stable(unstable) behaviour of the mode. Inhomogeneity therefore disrupts the balance of energy transfers occurring within a mode and this can precipitate instability. For both of the plates with spatially varying flexural rigidity, an increase in the divergence-onset value of $\Lambda^{F}$ was noted. Divergence onset occurs when the fluid forces due to the hydrodynamic stiffness exactly balance the restorative structural forces in the plate. The phase misalignment between the hydrodynamic-stiffness and the plate-deflection signals caused by the material inhomogeneity then requires a higher flow speed (for a given set of properties) to destablise the plate through divergence.

We now apply a similar approach to understand the pre-divergence single-mode flutter identified in Figure 13b when a further restraint was added to the system. We recall that it was the third mode that became unstable. The third, neutrally-stable, mode for the standard plate at $\Lambda^{F}=25$ is shown in Figure 15a. The associated plot of Figure 15b shows that the signal of the total pressure is mainly orthogonal to that of the velocity (the zero of the horizontal axis). Exact orthogonality of $-\Delta p$ throughout $x / L$ would mean that zero energy transfer occurs at every point. However, this is not the case; $\pm \psi$ is seen, especially in the regions adjacent to the nodal points of the mode shape in Fig. 15a. In fact, these are not exact nodes at non-zero flow speed; animations reveal that the modal motion contains a form of constrained wave travel through its cycle. Thus, the phase departures mean that spatially-dependent energy transfer - to and from the panel - occurs through the cycle of oscillation,. However, the exact antisymmetry of relative phase of pressure about $x / L=0.5$, together with exact orthogonality at $x / L=0.5$ means that the localised energy transfers sum to zero over the entire panel to yield the global neutral stability of this mode. Introducing a hinge constraint at the location of the centre of upstream quasi-node of Figure 15a modifies the plate's Mode-3 response as demonstrated by Figures $15 \mathrm{c}$ and $15 \mathrm{~d}$, and noting that this mode is now unstable. Comparing these with Figures $15 \mathrm{a}$ and $15 \mathrm{~b}$, we first note that the imposition of an exact upstream node forces a modification to the mode shape in its vicinity while the downstream quasi-node remains largely unaffected. Contrasting the relative phase of pressure plots, a sharp change now occurs at the exact node as would be expected; this confirms that it is wave-travel in the unrestrained case that creates regions of non-orthogonal pressure loading. The key feature of Figure $15 \mathrm{~d}$ is that the relative phase of $-\Delta p$ is not anti-symmetric about $x / L=0.5$ and nor are each 

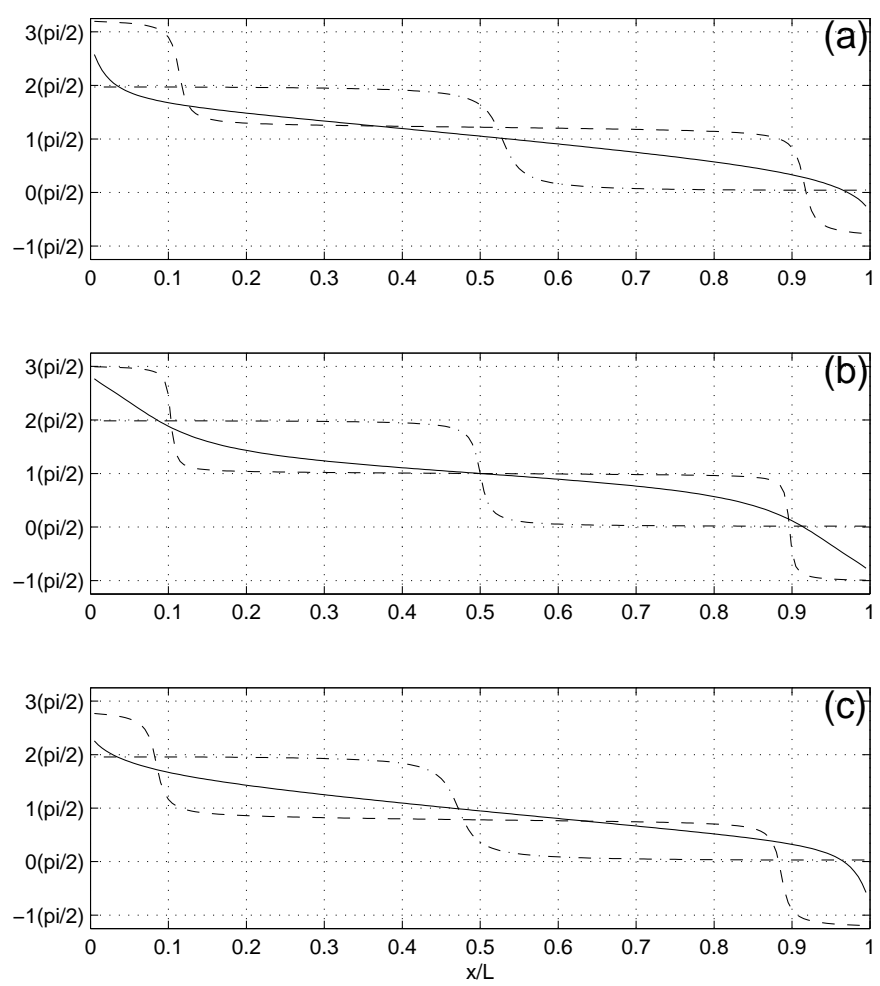

Figure 14. Variation along the plate of the phase of pressure loading relative to plate vertical velocity for Mode 1 at the pre-divergence flow speed corresponding to $\Lambda^{F}=25$. (a) decreasing (as in Figures 12a and 12b), (b) constant (as in Figures 3a and 3b), and (c) increasing (as in Figures 12c and 12d), flexural rigidity from plate leading edge: —, total pressure; - - - hydrodynamic-stiffness, and $-\cdot-$ hydrodynamic-damping components of total pressure.

of its constituent hydrodynamic stiffness and damping terms. The breaking of antisymmetry occurs principally in the region $x / L: 0.32 \rightarrow 0.5$. Thus, over one cycle, the sum, over the whole plate, of local irreversible energy transfers is positive and thus the mode is unstable.

Finally, the single-mode flutter that can result from spatial inhomogeneity in the present system is analogous to that which destablises short cantilevered-free flexible plates embedded in a unform flow; see, for example, Huang (1995), Guo \& Païdoussis (2000), Tang \& Païdoussis (2007) and Howell et al. (2008). In that system the divergence loop is stable because a plate with a free end cannot buckle and the spatially inhomogeneity required for the single-mode flutter is effectively provided by the very different boundary conditions at each end of the flexible plate.

\section{Conclusions}

A new method that fuses computational modelling and theoretical techniques for the direct determination of the eigenmodes of finite flow-structure systems has been developed. The method is readily adapted to take a Galerkin-type approach by rep- 

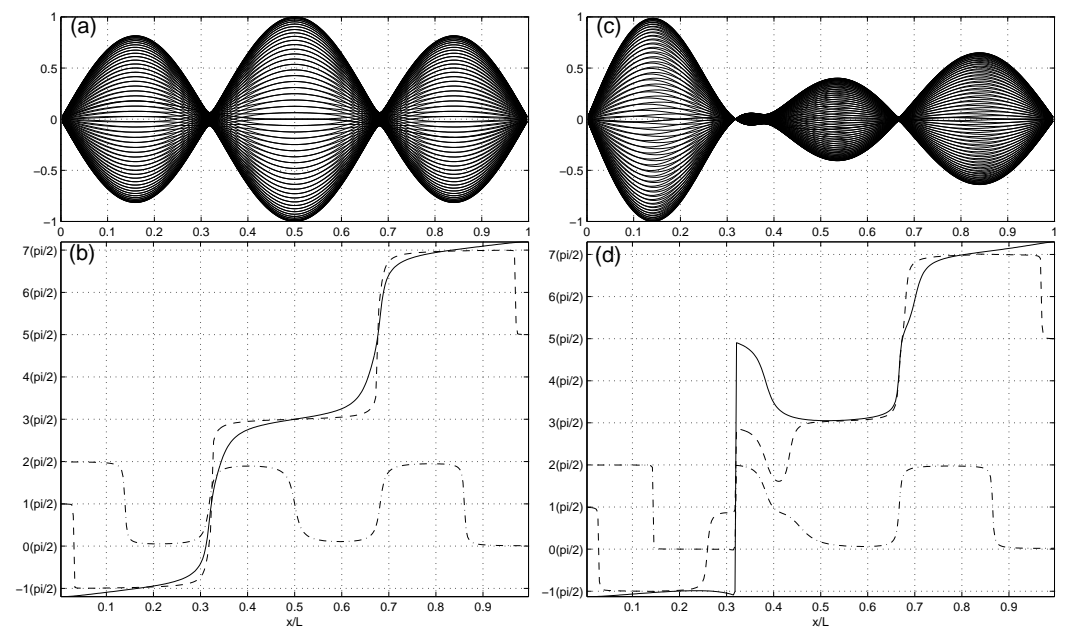

Figure 15. Third mode of an elastic plate without, (a) and (b), and with, (c) and (d), a hinged boundary constraint at $x / L=0.32$ at the pre-divergence flow speed corresponding to $\Lambda^{F}=25$ : (a) and (c) depict the eigenmodes, while (b) and (d) show the variation along the plate of the phase of pressure loading relative to plate vertical velocity where: total pressure; - - - hydrodynamic-stiffness, and $-\cdot-$ hydrodynamic-damping components of total pressure.

resenting deformations as a finite expansion of orthogonal in-vacuo modes of the structure. However, its greatest strength lies in the analysis of inhomogeneous systems and those with complex or multiple boundary conditions for which obtaining a suitable set of orthogonal functions would be problematical.

The method has been applied to the classical problem of a flexible panel, held at both its ends with hinged connections, interacting with a uniform flow. The eigenmodes extracted suggest that for realistic panels, modal-coalescence flutter is a continuous extension of the divergence instability that precedes it a lower flow speeds. For panels with structural damping, we show that there may exist a predivergence range of flow speeds in which all modes are attenuated. Unstable behavior would give the visual impression of sloshing waves, amplification accompanying the downstream motion and attenuation the upstream-directed motion. The speed of apparent travel of such motions increases as the applied flow speed is raised beyond the critical (divergence-onset) flow speed at which a static deformation exists. The behavior of a flexible plate with a continuous spring-foundation is shown to be far more complex in terms of participating modes although it is clearly shown that, overall, wall-dynamics are dominated by upstream-travelling (attenuating) and downstream-travelling (amplifying) motions.

In this paper, we have applied the direct method to perhaps the simplest flowstructure problem that could be framed and one that we show is amenable to accurate solution using a traditional Galerkin method. However, we have also demonstrated its use to conduct a linear stability analysis of flexible panels with spatially inhomogeneous wall properties and complex boundary conditions. In doing so we have discovered that material inhomogeneity or an added panel constraint, while postponing the divergence-onset flow speed, can introduce weakly unstable single- 
mode flutter behaviour into the system. This flutter is attributed to the disruption of the zero-sum balance of irreversible energy transfers that occurs within a mode in the pre-divergence range of flow speeds. While it would be attenuated, or even eliminated, by the effect of structural damping (which causes an irreversible energy flow out of the plate) it may still be of engineering significance for nearly elastic flexible plates.

The crux of the present method lies in the reduction of the flow-structure system to a single (matrix) equation for the interfacial variable. For a surface-based wall model (see Carpenter 1991 for a classification of flexible wall models) this is readily done for the structural side of the system. For ideal flow, the application of the divergence theorem to the Laplace equation reduces the flow solution to being surface based; i.e a reduction by one dimension that is a key advantage of the boundary-element method. Accordingly, any flow structure system in which ideal flow is assumed should be amenable to solution using the present method. This includes three-dimensional inviscid perturbations to a uniform flow over a flexible panel of finite width such as that studied by Lucey \& Carpenter 1993 (fixed edges) or Eloy et al. 2007 (cantilevered-free). Clearly, a system governed by one-dimensional perturbations in both flow and structure, such as the Bourrièrres and Roth models for a fluid-conveying flexible pipe (see Doaré \& de Langre 2006), could also be solved using the present methods. We also believe that our approach could be used for flow-structure systems that include a spatially varying mean flow, i.e. a flexible interface that is not uniformly aligned with the mean-flow direction, by extending the boundary-element method to compute additionally the mean-flow. Such a case could be considered as the fluid-side inhomogeneous problem having its structural parallel in the material inhomogeneity studied in this paper. Volume-based wall models (Carpenter 1991) are usually solved via a Helmholtz decomposition of the Navier equations (Duncan et al. 1986) in a travelling-wave approach or the finiteelement method in a discretised model. For the interaction of such a wall with an ideal flow, our approach is equally applicable, although computationally expensive since the single governing equation would be framed in the displacement of all the wall's collocation points.

The present use of potential flow also serves as the platform for the direct eigenvalue extraction of flow-structure systems with rotational and viscous effects included in the flow modelling. Recently, Ehrenstein \& Gallaire (2005) have used the direct extraction of eigenmodes to study the hydrodynamic stability of a laminar boundary layer over a rigid wall. The preliminary work of Pitman \& Lucey (2007) shows how a single system equation for the interfacial variable can be derived with the effect of a viscous boundary layer included. The flow solution is constructed using the present boundary-element method together with vortex elements governed by the two-dimensional linearised Navier-Stokes equations in velocity-vorticty form for the rotational part of the flow. Thereafter, both fluid-based and wall-based system eigenmodes are extracted. Finally, we note that the present derivation of a single homogenous differential equation for the coupled flow-structure system readily admits extension to the study of the same system responding to a forcing function that models a realistic initial-value problem. 


\section{Acknowledgements}

The authors are grateful to Professor P.W. Carpenter (University of Warwick) for a number of stimulating discussions on the topic of this paper. The authors also acknowledge the the Australian Research Council for their support under the Discovery Projects scheme.

\section{References}

Abrahams, I.D. \& Wickham, G.R. 2001 On transient oscillations of plates in moving fluids. Wave Motion 33, 7-23.

Carpenter, P.W. 1990 Status of transition delay using compliant walls. In: Viscous Drag Reduction in Boundary Layers, (eds. D.M. Bushnell and J.N. Heffner), AIAA, New York, 79-113.

Carpenter, P.W. \& Garrad, A.D. 1986 The hydrodynamic stability of flow over Kramertype compliant surfaces. Part 2. Flow-induced surface instabilities. Journal of Fluid Mechanics 170, 199-232.

Brazier-Smith, P.R. \& Scott, J.F. 1984 Stability of fluid flow in the presence of a compliant surface. Wave Motion 6(6) 547-560

Crighton, D.G. \& Oswell, J.E. 1991 Fluid loading with mean flow. I. Response of an elastic plate to localized excitation. Philosophical Transactions of the Royal Society of London A 335, 557-592.

Doaré, O \& de Langre, E. 2002 Local and global instability of fluid-conveying pipes on elastic foundations. Journal of Fluids and Structures 16(1), 1-14.

Doaré, O. \& de Langre, E. 2006 The role of boundary conditions in the instability of one-dimensional systems. European Journal Fluid Mechanics 25, 948-959.

Dugundji, J., Dowell, E. \& Perkin, B. 1963 Subsonic flutter of panels on a continuous elastic foundation. AIAA Journal 1, 1146-1154.

Duncan, J.H., Waxman, A.W. \& Tulin, M.P. 1985 The Dynamics of Waves between a Visco-Elastic Coating and a Fluid Flow. Journal of Fluid Mechanics, 158, 177-197.

Ehrenstein, U. \& Gallaire, F. 2005 On two-dimensional temporal modes in spatially evolving open flows: the flat-plate boundary layer. Journal of Fluid Mechanics 536, 209-218.

Ellen, C.H. 1973 The stability of simply supported rectangular surfaces in uniform subsonic flow. ASME: Journal of Applied Mechanics 95, 68-72.

Eloy, C., Souilliez, C. \& Schouveiler, L. 2006 Flutter of a rectangular cantilevered plate. Journal of Fluids and Structures 23, 904-919.

Garrad, A.D. \& Carpenter, P.W. 1982 A theoretical investigation of flow-induced instabilities in compliant coatings. Journal of Sound and Vibration 84(4), 483-500.

Guo, C.Q. \& Païdoussis, M.P. 2000 Stability of rectangular plates with free side-edges in two-dimensional inviscid channel flow. Journal of Applied Mechanics 67, 171-176.

Howell, R.M., Lucey, A.D., Carpenter, P.W. \& Pitman M.W. 2008 Interaction between a cantilevered-free flexible plate and ideal flow. Submitted to: Journal of Fluids and Structures (revised version submitted June 2008).

Huang, L. 1995 Flutter of cantilevered plates in axial flow. Journal of Fluids and Structures 9, 127-147.

Ishii, T. 1965 Aeroelastic instabilities of simply supported panels in subsonic flow. AIAA Paper AIAA-1965-752.

Lucey, A.D. 1998 The excitation of waves on a flexible panel in a uniform flow. Philosophical Transactions of the Royal Society of London A 356, 2999-3039.

Lucey, A.D. \& Carpenter, P.W., 1992. A numerical simulation of the interaction of a compliant wall and an inviscid flow. Journal of Fluid Mechanics 234, 121-146. 
Lucey, A.D. \& Carpenter, P.W. 1993 The hydroelastic stability of three-dimensional disturbances of a finite compliant panel. Journal of Sound and Vibration 165(3), 527-552.

Lucey, A.D., Cafolla, G.J. Carpenter, P.W. \& Yang, M. 1997 The nonlinear hydroelastic behaviour of flexible walls. Journal of Fluids and Structures 11, 717-744.

Lucey, A.D., Sen, P.K. \& Carpenter, P.W. 2003 Excitation and evolution of waves on an inhomogeneous flexible wall in a mean flow. Journal of Fluids and Structures 18(2), 251-267

Païdoussis, M.P. 1998 Fluid-Structure Interactions, Slender Structures and Axial Flows, Volume 1. Academic Press

Peake, N. 1997 On the behaviour of a fluid-loaded cylindrical shell with mean flow. Journal of Fluid Mechanics 338, 387-410.

Peake, N. 2004 On the unsteady motion of a long fluid-loaded elastic plate with mean flow. Journal of Fluid Mechanics 507, 335-366.

Pitman, M.W. \& Lucey, A.D. 2007. Eigen-analysis of a fully viscous boundary-layer flow interacting with a finite compliant surface. In Proc. of the 16th Australasian Fluid Mechanics Conference, 2nd-7th December 2007, Gold Coast, Australia (Eds. P. Jacobs, T. McIntyre, M. Cleary, D. Buttsworth, D. Mee, R. Clements, R. Morgan \& C. Lemckert) CD-ROM.

Tang and Païdoussis 2007. On the instability and the post-critical behaviour of twodimensional cantilevered flexible plates in axial flow. Journal of Sound and Vibration 305, 97-115.

Weaver, D.S. \& Unny, T.S. 1971 The hydroelastic stability of a flat plate. ASME: Journal of Applied Mechanics 37, 823-827. 